\title{
Pseudo-Césaire, Anastase, Pseudo-Athanase : quelques réflexions sur les collections de Questions-et-Réponses dans la littérature grecque chrétienne ${ }^{1}$
}

\author{
Caroline Macé \\ Goethe-Universität Frankfurt am Main, Germany
}

\begin{abstract}
Résumé. Le genre des questions-et-réponses dans la littérature grecque chrétienne se laisse mieux comprendre si l'on le définit comme une série de questions-et-réponses, présentées comme telles (et non comme des lettres ou des dialogues, par exemple) abordant des sujets variés et qui ne se réduisent pas à une seule catégorie de contenu (exégèse biblique ou explications scientifiques, par exemple). Ainsi restreint, le genre des questionset-réponses dans la littérature grecque chrétienne connaît sa période la plus faste aux VeVIIIe s. dans des milieux monastiques ouverts sur les problèmes et les interrogations du monde. Ce genre, d'une grande souplesse et d'une grande vitalité, permet de traiter des questions d'une façon plus accessible et plus libre qu'il ne serait possible de le faire dans une homélie ou un traité théologique.
\end{abstract}

\begin{abstract}
Pseudo-Caesarius, Anastasius, Pseudo-Athanasius: Some Thoughts on the Collections of Questions-and-answers in Greek Christian literature. The genre of questions-and-answers in Greek Christian literature is easier to understand if one defines it as a series of questions-and-answers, presented as such (and not as letters or dialogues, for example) dealing with a large variety of topics, which cannot be reduced to one single category of contents (biblical exegesis or scientific explanations, for example). So defined, the genre of questions-and-answers in Greek Christian literature is at its peak in the $5^{\text {th }}-8^{\text {th }}$ cent. in monastic milieus, open to worldly problems and questioning. This genre, with its great flexibility and vitality, allows for the handling of questions in a more accessible and free fashion than would have been possible in a homily or in a theological treatise.
\end{abstract}

\section{Introduction : y a-t-il un genre des questions-et-réponses ?}

Cette étude se concentre sur trois collections de questions-réponses [QR] écrites en grec entre 550 et 750, elles ont reçu dans la CPG les numéros 7482 (Pseudo-Césaire, ed. Riedinger 1989), 7746 (Anastase le Sinaïte, ed. Munitiz 2006) et 2257 (Pseudo-Athanase, PG Vol. 28, Col. 597-700). Si les deux dernières sont régulièrement associées et comparées, il est plus rare de les voir confrontées toutes

\footnotetext{
${ }^{1} \mathrm{Je}$ remercie Alain Bernard et Bernard Vitrac pour leur lecture attentive de cet article et leurs nombreuses propositions d'amélioration, que j'ai essayé de suivre autant que possible.

This is an Open Access article distributed under the terms of the Creative Commons Attribution License 4.0, which permits unrestricted use, distribution, and reproduction in any medium, provided the original work is properly cited.
} 
les trois ensemble ${ }^{2}$. Or, ces trois collections sont peut-être les représentants les plus « authentiques »du genre, difficile à cerner, des QR dans la littérature grecque chrétienne ${ }^{3}$.

La question du genre se pose de manière aiguë pour les collections de questions-réponses [QR] dans la littérature grecque chrétienne, comme l'a déjà souligné, après d'autres, C. Zamagni : « il est clair qu'il est presque impossible d'arriver à une définition de ce genre littéraire simple et claire, qui puisse rendre compte du large éventail d'où rayonne une quantité formidable de textes disparates. (...) il s'agit d'un genre très élastique » ${ }^{4}$. Claudio Zamagni avance une partie de la solution, en proposant de distinguer le genre littéraire proprement dit du procédé littéraire ${ }^{5}$. En effet, certains chercheurs ont eu tendance à intégrer dans la catégorie des $\mathrm{QR}$ des productions littéraires qui, certes, utilisent le procédé de la $\mathrm{QR}$, mais ressortissent nettement à d'autres genres ${ }^{6}$. Selon M. Mullett, un genre littéraire suit deux axes : la forme (qui induit la manière dont le texte sera réalisé - «performance » - et reçu) et le type (déterminé par l'occasion) ${ }^{7}$. D'une manière plus simple, plus traditionnelle, mais peut-être plus efficace, on pourrait dire que le genre se situe au croisement entre une forme et un contenu. Il est certainement difficile d'identifier un contenu commun à toutes les collections de $\mathrm{QR}$, et la forme de la $\mathrm{QR}$ elle-même peut prendre différents visages, comme nous le verrons. D'autre part la genèse et le développement de ce genre, depuis la littérature hellénistique jusqu'à la fin de la littérature byzantine, ne sont pas non plus faciles à définir ${ }^{8}$.

Dans cette problématique, le fait d'étudier ensemble les trois collections envisagées dans cet article peut apporter, je pense, un éclairage nouveau, pour les raisons suivantes : tout d'abord elles se présentent comme des collections de QR et rien d'autre - nous allons voir que dans certaines collections, les QR sont mises en scène comme des lettres ou comme des dialogues. Ensuite, il ne s'agit pas de florilèges sous forme de $\mathrm{QR}$ ou d'exégèse sous forme de $\mathrm{QR}$, mais ces collections présentent un contenu, ou plutôt une variété de contenus, qui leur est propre et qui ne se retrouve exactement tel quel dans aucun autre genre. Enfin, comme l'a souligné Gilbert Dagron, même si le genre des QR ne naît pas au V s. et ne meurt pas au $\mathrm{VIII}^{\mathrm{e}}$ s., il connaît un renouveau et un point culminant à l'époque des trois collections étudiées (il faut noter que Dagron ne considère pas la collection du Pseudo-Césaire dans son article $)^{9}$. En outre, Dagron a plusieurs remarques qui me semblent tout à fait pertinentes, mais qui ont rarement été considérées par la recherche sur le genre des QR, sans doute parce qu'elles se trouvent dans un article consacré à l'hagiographie - c'est pourquoi je me permets de les citer ici : « la littérature des Erôtapocriseis (...) a cette particularité, rare dans la littérature byzantine, de mêler théologie et discours «moral », et de donner la parole, fût-ce pour le temps d'une brève question, à ceux qui doutent et s'interrogent. Si fictive que soit la question, et si traditionnellement répétée I que soit la réponse, elles placent au cœur d'un vrai problème, au moins tant que le genre reste vivant. (...) Je crois donc justifié de considérer les premiers recueils de Questions [ceux des $\mathrm{V}^{\mathrm{e}}-\mathrm{VII}^{\mathrm{e}} \mathrm{s}$.] comme un ensemble cohérent (...); et je voudrais montrer qu'en abordant de front certains sujets (médecine et guérison, prédiction et prophétie, causalité naturelle et intervention divine), les auteurs de ces Questions

\footnotetext{
2 Dans cette étude, il aurait été légitime d'intégrer également les Quaestiones ad orthodoxos du Pseudo-Justin (CPG 6285) et les Quaestiones et dubia de Maxime le Confesseur (VII' s.) (CPG 7689) (ed. Declerck, 1982) - c'est quelque chose qu'il faudra faire, en effet, si l'approche développée ici s'avère utile.

3 Voir, par exemple, Papadogiannakis 2006 et Rey 2004 pour une présentation générale des QR dans la littérature chrétienne.

4 Zamagni 2004, 10.

5 Zamagni 2004, 10-12 et 2013, 242-243.

6 Zamagni 2013, 242.

7 Mullett 1992, 235-236.

${ }^{8}$ Les deux articles de Zamagni ( 2004 et 2013) font le point sur cette question et discutent les recherches antérieures de Bardy, Dörries, Perrone et Garzya.

${ }^{9}$ Dagron 1981, 143 ; voir aussi Cameron 1992 et Cameron 2006. Par un autre chemin, Zamagni ( 2013) arrive à la conclusion que les QR ne sont pas encore un genre au Ve s.
} 
cherchent très consciemment à délimiter le rôle du saint, la définition du miracle et la validité du genre hagiographique $» 10$.

Si l'on considère avec Dagron que tel est le contenu propre des collections chrétiennes de QR, alors on peut exclure de ce genre les œuvres de contenu différent, qui ont adopté la forme de la QR. Je pense qu'une des difficultés rencontrées par la recherche dans la délimitation du genre des QR chrétiennes tient justement à ce que l'on n'a pas reconnu que la première de ses caractéristiques est sa variété de contenu : comme l'a dit Dagron, les collections de QR mêlent théologie et morale, exégèse et discours scientifique. Il s'agit d'un point important, car c'est précisément cette diversité de contenu qui confère à ce genre son caractère « vivant », l'apparence authentique du questionnement : les questions ne sont pas guidées ni reliées entre elles par le texte qui est commenté ni par l'objet naturel qui est étudié, mais par la liberté (même si elle n'est qu'apparente) et l'imagination de ceux qui se posent des questions (même s'il s'agit de personnes fictives).

Ainsi, si, comme le dit Zamagni, le premier auteur chrétien à avoir écrit une œuvre sous forme de QR est Eusèbe de Césarée ( $\mathrm{IV}^{\mathrm{e}} \mathrm{s}$.), dont nous avons conservé, en partie du moins, deux séries de QR sur les évangiles (CPG 3470) ${ }^{11}$, il s'agit là d'une œuvre exégétique sous forme de $Q R$ et non d'une collection de QR telle que nous l'entendons ici ${ }^{12}$. S'il est vrai que le procédé des QR a été adapté du contexte scolaire à la littérature, il n'est pas étonnant que l'exégèse ait été le genre par le biais duquel le procédé de la QR s'est frayé un chemin de la littérature philosophique tardo-antique (que l'on pense aux Quaestiones Homericae de Porphyre [III ${ }^{\mathrm{e}} \mathrm{s}$.] ou à son commentaire sur les Catégories d'Aristote sous forme de QR) à la littérature théologique chrétienne. Sur ce point, cependant, l'influence de la littérature juive hellénophone ne doit pas être négligée non plus : les QR, dans le cadre de l'exégèse de l'Ancien Testament, sont un genre bien représenté, notamment par Philon d'Alexandrie ( $\mathrm{I}^{\mathrm{e}}$ s. av. - $\mathrm{I}^{\mathrm{e}}$ s. ap. JC), mais, d'après P.W. van der Horst, il aurait été précédé au $\mathrm{III}^{\mathrm{e}} \mathrm{s}$. av. JC par Démétrius le chronographe, dont Eusèbe et Clément d'Alexandrie nous ont conservé des fragments ${ }^{13}$. Le degré minimal de l'exégèse et un aspect du caractère didactique de la littérature associée aux QR sont illustrés par une ouvre, peu souvent discutée, qui elle aussi prend la forme de QR, l'Hypomnesticon de Joseph de Tibériade (PG vol. 106, col. 15-176), que S. Mimouni date du IV ${ }^{\mathrm{e}}$ s. et dont il conteste le rattachement au genre des $\mathrm{QR}$, affirmant qu'il est vain de chercher à ranger l'Hypomnesticon dans un genre littéraire, car il est pratiquement unique en son genre ${ }^{14}$. Cette œuvre contient 167 « chapitres », en réalité des listes de personnages (prêtres, rois etc.) ou phénomènes remarquables (mois hébreux, hérésies etc.) en relation avec l'Ancient ou le Nouveau Testament.

Le second domaine dans lequel on remarque la prépondérance du milieu scolaire et l'influence de la littérature tardo-antique est celui des sciences et de la médecine : les Problemata attribués à Aristote ${ }^{15}$ en sont un bon exemple, mais il existe aussi, dès l'antiquité tardive, des recueils de QR à caractère « scientifique », notamment dans le domaine médical ${ }^{16}$. Cette utilisation des QR dans la littérature scientifique perdure à Byzance, par exemple dans les Quaestiones Physicae de Théophylacte Simocatta $\left(\right.$ VII ${ }^{\mathrm{e}}$ s.) (ed. Massa Positano, 1965) ${ }^{17}$.

\footnotetext{
10 Dagron 1981, 143-144.

11 Zamagni 2004, 7. Ces deux séries sont conservées partiellement par un épitomé édité par C. Zamagni en 2009 (Sources chrétiennes 523).

12 De même, par exemple, les Quaestiones in loca difficilia scripturae sacrae de Théodoret de Cyr (Ve s.) (CPG 6200-6201).

13 van der Horst 2004, 55-57.

14 Mimouni 1997 [2010], 257. Pour une tentative d'éclaircissement sur le terme ú $\pi$ $\mu \nu \eta \mu \alpha$ dans les titres d'œuvres byzantines, voir Gribomont 2012, et aussi Durst 1989.

15 Voir Blair 1999, De Leemans - Goyens 2006.

16 Voir les Quaestiones medicinales de Rufus d'Éphèse ( $\mathrm{I}^{\mathrm{e}}-\mathrm{II}^{\mathrm{e}} \mathrm{s}$.) (ed. Gärtner, 1962) et de Cassius le iatrosophiste (II ${ }^{\mathrm{e}}-\mathrm{III}{ }^{\mathrm{e}}$ s.) (ed. Ideler, 1841); cf. Ieraci Bio, 1995.

17 Chrysos 2002, 17-18 fournit une liste, basée sur le manuel classique de H. Hunger, de recueils de QR dans la littérature « séculaire » savante.
} 
Enfin, dans le domaine moral, on doit citer l'Asceticon magnum de Basile de Césarée (IV ${ }^{\mathrm{e}} \mathrm{s}$.) (CPG

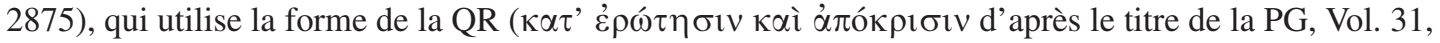
Col. 905), mais qu'on ne rangera pas dans le genre des QR car son contenu est strictement ascétique.

Le caractère didactique de la $\mathrm{QR}$, comme procédé littéraire, demeure prédominant à Byzance, même en dépit de l'influence de la scholastique latine ${ }^{18}$. Ce caractère est évidemment présent aussi dans les collections de QR envisagées dans cet article. Ces collections ont pour caractéristique, comme l'a souligné Dagron, de traiter aussi bien de questions d'exégèse que de préoccupations d'ordre scientifique ou médical (même si c'est à un niveau très élémentaire), ou de questions de morale.

L'autre caractéristique du genre des QR évoquée plus haut est le fait que le procédé de la QR est bien présenté comme la forme principale du texte, à l'exclusion d'autres formes. Certaines collections de lettres sont parfois considérées comme des collections de $\mathrm{QR}$, alors que la mise en scène littéraire propre à la lettre est différente de celle de la $\mathrm{QR}$, et ce même quand la lettre répond explicitement à une question posée préalablement. Ainsi dans la collection de lettres de Barsanuphe et de Jean de Gaza (VI ${ }^{\mathrm{e}} \mathrm{s}$ ) (ed. Neyt - de Angelis-Noah 1997-2002), la question posée par les moines est chaque fois résumée avant la réponse écrite. Le titre latin traditionnel, ayant une certaine autorité générique, donné à cette œuvre dans la CPG (7350) est seulement « quaestiones et responsiones », tandis que dans les deux titres grecs édités par Chitty (1966, 456 [12]), le double caractère de lettre et de QR est clairement exprimé : « غ̇ं $1 \sigma \tau 0 \lambda \alpha i ̀$

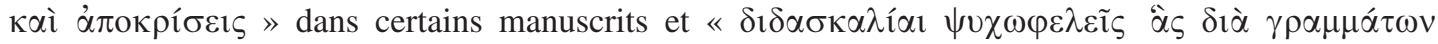

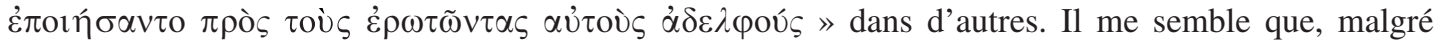
certaines similarités de conventions entre les deux genres, collections de lettres et collections de QR, il est plus judicieux de considérer comme ressortant au genre épistolographique ${ }^{19}$ ce qui clairement se présente comme tel.

Une autre façon de mettre en scène les QR est la forme du dialogue, le plus souvent fictif, entre des personnages, tantôt historiques, comme Grégoire de Nazianze et Basile de Césarée, par exemple ${ }^{20}$, tantôt typologiques ou archétypiques, comme le juif et le chrétien, le païen et le chrétien, etc. Là encore, les conventions du genre, même si elles restent proches, sont différentes ${ }^{21}$.

Il faut dire encore quelques mots à propos de deux œuvres que Dagron (et d'autres) considère comme des collections de QR : les Amphilochia de Photius (IX ${ }^{\mathrm{e}}$ s.) (ed. Laourdas - Westerink, 1986-1987) et les « Apories » de Michel Glycas (XII ${ }^{\mathrm{e}}$ s.) (ed. Eustratiades, 1906-1912). Dagron écrit au sujet de ces deux œuvres : «à partir du IX ${ }^{\mathrm{e}}$ s., les $\mathrm{QR}$ se gèlent en florilèges » ${ }^{22}$. Tout d'abord, il faut objecter à ce jugement de Dagron que l'existence d'un florilège sous forme de QR dès le VI ${ }^{\mathrm{e}}$ s., celui d'Etienne Gobar, préservé de façon très abrégée dans la Bibliothèque de Photius (codex 232 - ed. Henry, 1959-1977) ${ }^{23}$, montre que, dès le début, les réponses, dans ces collections, pouvaient être soit « originales », soit puiser plus ou moins largement, voire totalement, dans la littérature antérieure. Cet usage des citations, si répandu à Byzance ${ }^{24}$, est, je pense, un autre aspect du caractère didactique des collections de QR. Comme nous le verrons, le Pseudo-Césaire cite de nombreuses sources dans ses QR, mais on ne peut toutefois pas qualifier son œuvre de florilège, comme c'est le cas pour Etienne Gobar. De ce point de vue, tant les Amphilochia de Photius que les Apories de Glycas (ces deux œuvres s'occupant d'ailleurs principalement d'exégèse biblique) ne peuvent pas non plus être rangées purement et simplement parmi les florilèges, même si la majorité du texte de Glycas n'est pas originale (il cite notamment des

\footnotetext{
18 Demetracopoulos 2012, 336 : "the traditional Byzantine quaestio pertained to teaching, whereas the Latin quaestio pertained to research".

19 Ce genre est lui-même difficile à définir : voir Hatlie 1996, Mullett 2008, Papaioannou 2010.

${ }^{20}$ Heinrici 1910 \& 1911.

21 Voir par exemple Cameron 1991, Lim 2008

22 Dagron 1981, 144.

23 Sur ce florilège dogmatique, voir Bardy 1947.

24 Odorico 1990 et 2011.
} 
collections de QR d'autres auteurs), et même si, sur les 300 questions adressées à Photius, près de la moitié des réponses proviennent soit d'autres œuvres de Photius lui-même, soit d'auteurs antérieurs ${ }^{25}$. Quoiqu'il en soit, le fait qu'il s'agit de collections de lettres, comme l'a bien montré E.-S. Kiapidou à propos de Glycas $^{26}$, ne permet pas d'intégrer ces deux œuvres parmi les collections de QR proprement dites.

Si les collections de QR dans la littérature chrétienne grecque nous intéressent dans le cadre de ce volume collectif, c'est spécialement à cause de l'organisation de ces QR en séries plus ou moins cohérentes, voulues ou non par leur auteur. L'apparence de spontanéité des questions (ce qui n'implique pas, comme nous allons le voir, que les réponses soient totalement libres), traitant de tous les sujets, sans être liées à un texte-source à commenter où à un domaine du savoir délimité, rend cette organisation potentiellement très ouverte. On observe toutefois dans les collections de $\mathrm{QR}$ un certain ordre, qui n'est pas toujours facile à discerner, qui n'est pas toujours évident, et qui a dès lors connu des fluctuations dans les traditions manuscrites respectives de ces textes.

\section{Proposition de critères d'appartenance générique et application au corpus}

Dans ce qui suit, je me propose d'examiner trois collections typiques de $\mathrm{QR}$ datant du $\mathrm{VI}^{\mathrm{e}}$ au $\mathrm{VIII}^{\mathrm{e}}$ s. en utilisant une grille d'analyse qui mette en relief les caractéristiques communes et les différences entre ces trois collections. La grille d'analyse comprend cinq éléments : (1) la question de l'auteur ou de l'autorité, (2) le titre, (3) le type de contenu et les sources utilisées, (4) les principes d'organisation et de cohérence, (5) la diffusion et la réception de l'œuvre.

\subsection{Auteur / autorite}

Contrairement à la collection d'Anastase (mais la situation est plus complexe qu'il n'y paraît au premier abord), les collections de QR attribuées à Athanase et à Césaire sont pseudonymiques et cette «pseudonymité » ne semble pas accidentelle, car elle est solidement ancrée dans la tradition manuscrite de ces œuvres. Est-il possible de comprendre pourquoi ces deux collections ont été attribuées (par leur auteur lui-même ou par le milieu dans lequel ces œuvres ont été composées) respectivement à Athanase d'Alexandrie ( $\mathrm{IV}^{\mathrm{e}} \mathrm{s}$.) et au frère de Grégoire de Nazianze ( $\mathrm{IV}^{\mathrm{e}} \mathrm{s}$.) ?

Si de nombreuses œuvres ont été attribuées à Athanase dans la littérature byzantine, l'attribution à Césaire est un cas isolé. En outre, comme l'a fait remarquer I. Perczel ${ }^{27}$, les informations concernant Césaire dans le titre de la collection de QR (voir plus loin) ne correspondent pas à ce que l'on sait du Césaire historique grâce à l'éloge, bien connu ${ }^{28}$, que Grégoire de Nazianze a écrit de lui, à savoir qu'il était un médecin laïc à la cour impériale, et non un enseignant versé dans la théologie, et qu'il était resté seulement quelques années à Constantinople avant de mourir à Nicée des suites de blessures reçues lors du tremblement de terre de 368 (Discours 7, Sect. 15, ed. Calvet-Sébasti 1995, 217). Si l'on ne peut totalement exclure la possibilité que la mention de la fraternité avec Grégoire de Nazianze ait pu être ajoutée postérieurement, et que donc l'identification de Césaire, auteur de ces QR, avec Césaire, le frère de Grégoire, soit fortuite, il semble plus probable que l'auteur de ces QR ait vraiment voulu les placer sous le nom de Césaire, le frère de Grégoire, ce qui est un patronage pour le moins curieux, car Césaire

\footnotetext{
25 Constas 1999, 99-100.

${ }^{26}$ Kiapidou 2013. Dans cet article, Kiapidou note que le titre donné par l'éditeur au recueil de lettres de Glycas, à savoir « E’s

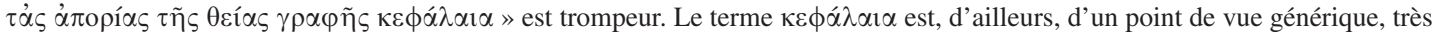
problématique : cf. De Ridder - Levrie (sous presse).

27 Perczel 2006, 72.

28 Calvet-Sébasti 1995, 41.
} 
ne peut guère être considéré comme une « autorité ». Plusieurs tentatives ont été faites pour identifier l'auteur de cette collection, dont la langue maternelle n'était vraisemblable pas le $\operatorname{grec}^{29}$. Riedinger (1969) en fait un moine du monastère des Acémètes à Constantinople, vivant au milieu du $\mathrm{VI}^{\mathrm{e}} \mathrm{s}$. Si personne n'a élevé de critiques contre la datation de l'œuvre par Riedinger, la personnalité de l'auteur de ce texte reste un mystère ${ }^{30}$.

La collection du Pseudo-Athanase est certainement antérieure à 750, parce qu'elle est citée, sous le nom d'Athanase et avec le titre qu' on lui connaît par la tradition directe (voir plus loin), dans la Doctrina Patrum et dans un florilège de Jean Damascène ${ }^{31}$. Elle est généralement datée du début du VII ${ }^{\mathrm{e}} \mathrm{s}$. et son origine est située en Palestine ${ }^{32}$. L'identité de son auteur est inconnue, mais la proximité linguistique et thématique de cette œuvre avec la collection de QR d'Anastase est telle qu'on peut les supposer toutes deux issues du même milieu. À la question, depuis longtemps débattue ${ }^{33}$, de savoir laquelle de ces deux collections a influencé l' autre, D. Krausmüller ( 2013) répond qu'elles semblent réagir, indépendamment l'une de l'autre, au même ouvrage présentant une vue radicale sur la résurrection de la chair, ouvrage qui n'a pas été conservé ${ }^{34}$.

La collection de 103 QR, que J. Munitiz a éditée comme étant la collection « authentique » des QR d'Anastase ${ }^{35}$, est-elle vraiment d'Anastase le Sinaïte ? Personne n'a élevé de doute à ce sujet et les allusions nombreuses aux Arabes dans les QR pointent en effet vers une origine égypto-palestinienne et vers la seconde moitié du VII ${ }^{\mathrm{e}} \mathrm{s} .{ }^{36}$ Munitiz pense que la collection a été compilée après la mort d'Anastase, ce qui expliquerait son apparent désordre ${ }^{37}$. Il faut noter cependant que l'absence d'un ordre immédiatement visible pour ce genre de collection de QR n'a rien de surprenant (voir plus loin). Il est toutefois frappant que la collection n'ait été conservée dans aucun manuscrit ancien du monastère du Sinaï.

Quoi qu'il en soit, ces trois collections semblent issues de milieux monastiques.

\subsection{Titre}

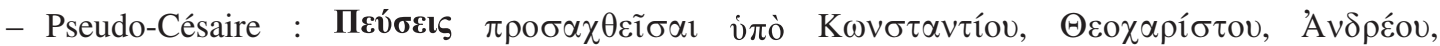

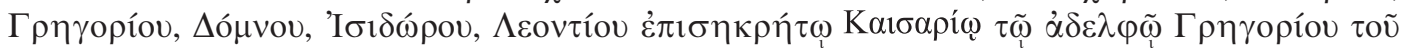

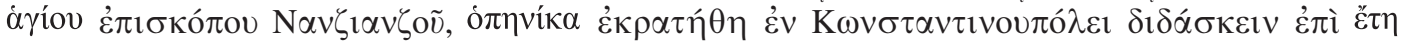
Eǐ́cođıv - «Questions posées par Constantin, Théocariste, André, Grégoire, Domnus, Isidore, Léonce, à l'épisècrètos Césaire, le frère de Grégoire le saint évêque de Nazianze, alors qu'il est resté enseigner à Constantinople pendant 20 ans ${ }^{38}$.

\footnotetext{
${ }^{29}$ Lackner ( 1991) a montré qu'il s'agit d'une explication convaincante au fait que le texte présente à la fois des tournures peu correctes et des mots rares, dont certains s'expliquent par l'utilisation de dictionnaires ou de lexiques.

${ }^{30}$ Perczel (2006) l'identifie avec Théodore Ascidas, un crypto-Origéniste, mais cela ne convainc guère : Papadogiannakis 2013, 274 n.12. Tzamalikos ( 2012) a proposé une autre identification avec Cassien le Sabaïte, mais l'argumentation, basée principalement sur des proximités de vocabulaire, est intenable.

31 Macé 2013, 128-143.

32 Haldon ( 1992, 120) écrit à propos de la QR 44 du Pseudo-Athanase: "[it] refers to the fact that the Arians had held Palestine and the Holy Places for only a short time before they had been driven out, and that "barbarians" had often invaded the region in the past. This might refer to the Persian invasion and occupation of the period 614 to 626-27'. Bandini $(1997,185-186)$ arrive à la même conclusion.

33 Macé 2013, 143-149.

34 Indépendamment, sur la même thématique de la résurrection dans les collections d'Anastase, du Pseudo-Athanase et du PseudoJustin, voir Papadogiannakis 2014

35 Munitiz 2006.

36 Haldon 1992, 120-121.

37 Munitiz, 2006, L.

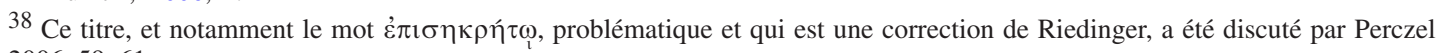
2006, 59-61.
} 


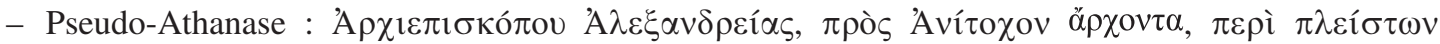

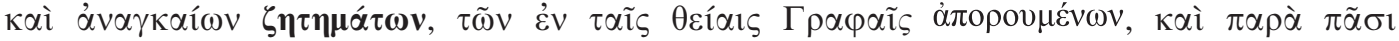

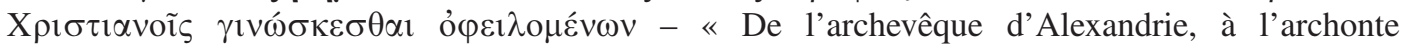
Antiochus, au sujet d'interrogations très nombreuses et nécessaires, de la part de ceux qui se posent des questions sur les saintes écritures, et qui sont utiles à savoir pour tous les chrétiens ».

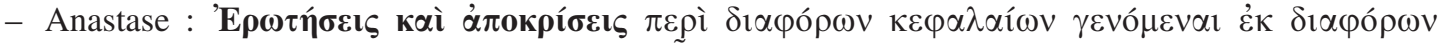

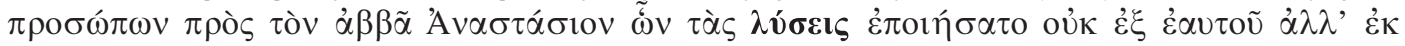
$\pi \mathrm{o} \lambda \lambda \tilde{\eta} \varsigma \pi \varepsilon i \rho \alpha_{\varsigma}$ - «Questions et réponses au sujet de différents sujets (chapitres) posées par différentes personnes à l'Abba Anastase pour lesquelles il a proposé les solutions non pas à partir de lui-même mais d'une longue expérience ».

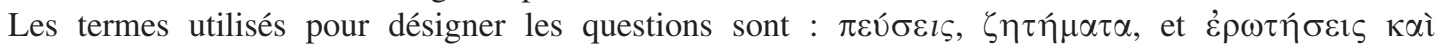

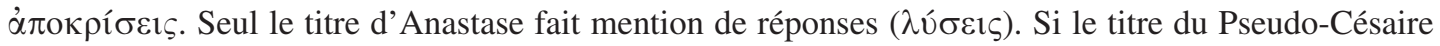
détaille les noms des différentes personnes censées avoir posé ces questions, en ce qui concerne la collection d'Anastase on sait seulement qu'elles étaient plusieurs. Seule la collection du PseudoAthanase a un dédicataire, Antiochus " archonte », à qui est aussi dédiée une autre œuvre pseudoathanasienne, la Doctrina ad Antiochum ducem (CPG 2255), dont la seconde partie (ch. 14-21) raconte la vie d'Antiochos, probablement un moine d'un monastère proche de St-Sabas en Palestine ${ }^{39}$. Il faut noter que, contrairement à ce qu'indique son titre, la collection pseudo-athanasienne ne traite pas seulement de questions exégétiques.

\subsection{Contenu et sources}

La collection de QR du Pseudo-Césaire contient 218 QR. Riedinger (1969) a montré qu'elle utilise et cite souvent littéralement et parfois longuement (mais sans les nommer) de nombreuses sources. La part d'originalité du Pseudo-Césaire est donc réduite, sans qu'il s'agisse cependant à proprement parler d'un florilège. La dernière $\mathrm{QR}$ de la collection est un pamphlet anti-judaïque, tout comme la dernière $\mathrm{QR}$ du Pseudo-Athanase.

La collection du Pseudo-Athanase contient 137 QR dans la version éditée dans la PG (vol. 28, col. 597-700), et beaucoup de manuscrits présentent à peu près ces $137 \mathrm{QR}^{40}$. Toutefois, Jean Damascène († ca. 750) connaissait une collection de 100 QR seulement ${ }^{41}$. La QR 137 (polémique anti-judaïque) et la QR 136 (sur la conversion des païens) semblent ne pas appartenir à la collection originelle, pour différentes raisons, mais leur insertion à l'intérieur de celle-ci doit cependant remonter assez haut ${ }^{42}$.

La collection d'Anastase compte, comme nous l'avons dit plus haut, 103 QR. Toutefois, en appendice, Munitiz édite 29 autres QR, qui sont elles aussi régulièrement attribuées à Anastase, mais qui se trouvent soit dans un autre type de collection, soit comme QR isolées dans différents manuscrits. Cela montre que le problème du contenu du corpus anastasien et de son authenticité est complexe et n'a pas été entièrement résolu par Munitiz. Pas plus que les QR du Pseudo-Athanase, celles d'Anastase ne montrent un usage extensif de sources antérieures, comparable au Pseudo-Césaire.

Dagron $(1981,144)$ note que les QR à contenu médical chez Anastase ont été considérées comme une interpolation par certains savants. Mais un contenu médical se retrouve également dans les deux autres collections, notamment chez le Pseudo-Césaire, et la médecine devait faire partie, jusqu'à un certain point, de la formation de certains moines $^{43}$.

\footnotetext{
39 Bandini 1997, 181 et 184-185.

40 En l'absence d'une édition critique il est impossible de fournir ici autre chose que des impressions, basées sur l'examen d'un petit nombre de témoins.

41 Macé 2013, 128.

42 Macé - De Vos 2013.

43 Congourdeau 2005, Macé 2014.
} 


\section{SHS Web of Conferences}

\subsection{Organisation en un ensemble}

Contrairement aux deux autres collections, les QR sont précédées chez le Pseudo-Césaire d'une sorte de prologue, très rhétorique, lui aussi sous forme de $\mathrm{QR}$, où les interlocuteurs de Césaire lui demandent comme à un «père qui aime ses enfants » $(\varphi \imath \lambda o ́ \tau \varepsilon \kappa v o \varsigma \pi \alpha ́ \tau \eta \rho)$ de leur offrir une guidance spirituelle au sujet de la sainte trinité et des saintes écritures, à eux « qui naviguent à travers la mer démontée

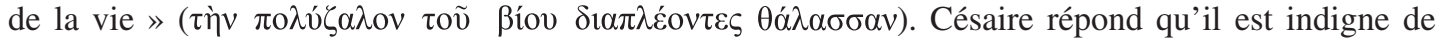
cette tâche, mais, après une longue captatio benevolentiae, il accepte néanmoins : " Interrogez-moi donc, admirables amis, sur les sujets qui vous plaisent, afin de récolter les germes divins de ceux-ci à

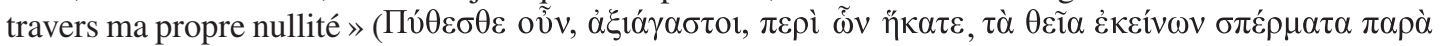

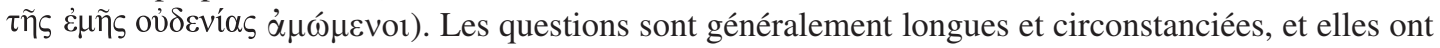
été visiblement rédigées par la même personne que celle qui a composé les réponses. La « mise en scène » littéraire est de toute évidence plus soignée chez le Pseudo-Césaire que dans le cas des deux autres collections (cette conscience de genre est, soit dit en passant, un peu paradoxale, si l'on considère que la collection du Pseudo-Césaire est la plus ancienne). Le titre et le prologue de la collection lui donnent une légitimité et une raison d'être : le pseudo-Césaire a écrit cette œuvre parce que plusieurs personnes l'en avaient instamment prié. L'ordre dans lequel les questions sont posées est, dans cette mise en scène, dû à ceux qui l'interrogent. En réalité il est bien clair que l'ordre de l'exposé est voulu par l'auteur et donc le caractère de fiction littéraire de ces QR est bien évident pour n'importe quel lecteur.

Chez le Pseudo-Césaire, un pinax, qui semble bien remonter à l'auteur ou en tout cas à l'archétype de toute la tradition, donne la liste des sujets envisagés dans chaque QR. Comme les questions, sont longues, le sujet sur lequel elles portent n'est pas toujours évident à identifier en première lecture, et le pinax permet donc une navigation plus rapide à travers les QR, qui sont numérotées dans les manuscrits et dans le pinax. Le pinax nous permet de constater que l'ordre des QR n'est pas du tout aléatoire, mais qu'il suit une progression logique, de Dieu à sa création et au salut de celle-ci : Dieu $>$ monde $>$ être humain > Dieu incarné. En effet, les QR 2-49 concernent les Personnes de la Trinité et les anges ; les QR 50-117 traitent de la création du monde visible ; les QR 121-185 se concentrent sur la création de l'homme et le paradis ; enfin, les QR 186-218 discutent de l'incarnation du Christ et commentent des passages du Nouveau Testament. À l'intérieur de chaque grande section, on pourrait encore détailler quelques sous-sections : les QR 140-158, par exemple, qui ne sont d'ailleurs pas des QR (en tout cas il n'y a pas de question exprimée), détaillent chacune des parties du corps humain, littéralement de la tête aux pieds.

Les QR sont numérotées aussi dans les collections d'Anastase et du Pseudo-Athanase, du moins dans certains manuscrits, mais on ne trouve généralement pas de pinax. Si l'ordre des QR dans ces deux collections n'est pas aussi clair que chez le Pseudo-Césaire, il n'est cependant pas totalement absent, spécialement chez le Pseudo-Athanase, où l'on trouve aussi une sorte de rangement plutôt associatif : les premières QR portent sur le baptême, puis on passe aux anges, aux démons, à la création, à des questions relatives à l'exégèse, au péché etc. Comme nous l'avons dit plus haut Munitiz (2006, L) considère le désordre de la collection de QR d'Anastase comme un indice du fait que cette collection ait pu être compilée par ses disciples après sa mort. L'argument n'est pas très probant, étant donné que le genre n'appelle pas en soi un ordre strict. L'hypothèse avancée par Munitiz pose toutefois la question de la possible forme d'existence, de conservation, voire de circulation, des QR avant leur intégration dans une collection. L'idée que certaines QR ont pu être transmises, sous forme orale ou écrite, de façon indépendante ou par petits groupes, avant d'être rassemblées dans une collection, est purement théorique, me semble-t-il, et nous n'en avons aucune trace. Au contraire, l'organisation de la collection du Pseudo-Césaire, avec son prologue et son pinax, pointe vers une origine livresque, de même que l'attestation à date ancienne (avant 750) de l'existence d'une collection de 100 QR du Pseudo-Athanase. 


\subsection{Diffusion et réception}

La tradition manuscrite de la collection du Pseudo-Césaire est relativement modeste si on la compare avec celle des deux autres collections, dont les manuscrits médiévaux se comptent par plusieurs dizaines. Trois manuscrits importants et anciens seulement nous ont conservé le texte du Pseudo-Césaire, les quelques autres manuscrits recensés par Riedinger (1969) sont récents. Cela pourrait témoigner d'une moindre popularité de la collection du Pseudo-Césaire, si celle-ci n'avait pas, comme les deux autres, fait l'objet de traductions médiévales et n'avait pas été citée par d'autres auteurs ${ }^{44}$.

À côté de ce qu'il appelle la collection « originale » des QR anastasiennes, Munitiz (2006) mentionne plusieurs autres types de collections. Parmi elles, la «collection a » est historiquement la plus importante. Il s'agit d'une collection de 154 QR éditées par Jacob Gretser en 1617 - cette édition a été reprise dans la PG (Vol. 89, Col. 312-824). Cette collection mêle des QR « authentiques » à une collection de $88 \mathrm{QR}$, inauthentique (mais qui utilise des QR authentiques d'Anastase). Cette collection contient un important florilège et elle a été traduite en slavon sur l'ordre de Syméon, qui devint roi de Bulgarie en $893^{45}$. Cette diversification des collections basées sur les QR d'Anastase atteste de la popularité et de la vitalité de ce texte. Malgré les recherches de Munitiz (2006), il resterait à éclairer davantage l'histoire des relations entre ces collections et de leur diffusion dans le temps et dans l'espace. L'histoire du texte des $\mathrm{QR}$ du Pseudo-Athanase, quant à elle, reste entièrement à écrire ${ }^{46}$. D'assez nombreux manuscrits contiennent à la fois les QR du Pseudo-Athanase et l'une des collections associées à Anastase.

Même si les QR semblent a priori passibles d'exister isolément et les collections d'être refondues, on voit que, plus souvent, la collection en tant que telle est recopiée et considérée comme une œuvre.

\section{Conclusion}

Les trois collections de QR connues sous les noms de Césaire, d'Anastase et d'Athanase sont exemplaires du genre des $\mathrm{QR}$ à l'époque de son efflorescence dans la littérature grecque chrétienne $\left(\mathrm{V}^{\mathrm{e}}\right.$ $\mathrm{VII}^{\mathrm{e}} \mathrm{s}$.). Toutes trois sont probablement issues de milieux monastiques, et elles montrent une largeur du champ intellectuel de leurs auteurs et par là de leurs lecteurs qui est assez remarquable. Cela explique sans doute le succès de ces collections, en tout cas de celles d'Anastase et du Pseudo-Athanase : elles offrent en condensé et dans une forme accessible, et sans doute moins exigeante pour le lecteur qu'un traité continu, ce qu'il est important de savoir non seulement à un moine mais plus largement à un chrétien à Byzance.

\section{Références}

Bandini 1997 = Bandini, Michele, "La doctrina ad Antiochum ducem pseudo-atanasiana. Tradizione diretta, struttura, datazione", Prometheus, 23 (1997), 171-187.

Bardy 1947 = Bardy, Gustave, «Le florilège d'Etienne Gobar », Revue des études byzantines 5 (1947), 5-30.

Blair 1999 = Blair, Ann, "The Problemata as a natural philosophical genre", in: A. Grafton - N. Saraisi (eds.), Natural Particulars: Nature and the Disciplines in Renaissance Europe, Cambridge (Mass.): MIT Press, 1999, 171-204.

\footnotetext{
44 Riedinger, 1969.

45 Sur cette collection en grec, voir De Groote 2015 ; sur l'importance de cette collection en slavon, voir Thomson 2014.

46 Voir néanmoins, pour une contribution limitée, De Vos sous presse.
} 
Bussières 2013 = Bussières, Marie-Pierre (ed.), La littérature des questions et réponses dans l'Antiquité profane et chrétienne: de l'enseignement à l'exégèse. Actes du séminaire sur le genre des questions et réponses tenu à Ottawa les 27 et 28 septembre 2009 (Instrumenta Patristica et Medievalia 64), Turnhout: Brepols 2013.

Calvet-Sébasti 1995 = Grégoire de Nazianze, Discours 6-12, ed. Marie-Ange Calvet-Sébasti (Sources Chrétiennes 405), Paris: Cerf, 1995.

Cameron 1991 = Cameron, Averil, "Disputations, Polemical Literature and the Formation of Opinion in the Early Byzantine Period", in: G. J . Reinink - H.L.J. Vanstiphout (eds.), Dispute Poems and Dialogues in the Ancient and Mediaeval Near East (Orientalia Lovaniensia Analecta 42), Leuven: Peeters, 1991, 91-108.

Cameron 1992 = Cameron, Averil, "New Themes and Styles in Greek Literature, $7^{\text {th }}$ and $8^{\text {th }}$ Centuries", in: Cameron - Conrad 1992, 81-105.

Cameron 2006 = Cameron, Averil, "New Themes and Styles in Greek Literature, A Title Revisited", in: Johnson 2006, 11-28.

Cameron - Conrad 1992 = Cameron, Averil - Conrad, L.I. (eds), The Byzantine and Early Islamic Near East. Problems in the Literary Source Material (Studies in Late Antiquity and Early Islam, I/1) Princeton: Darwin Press 1992.

Ceulemans - De Leemans sous presse = Ceulemans, Reinhart - De Leemans, Pieter (eds.), On Good Authority. Tradition, Compilation and the Construction of Authority in Literature from Antiquity to the Renaissance (LECTIO Studies on the Transmission of Texts and Ideas 3), Turnhout: Brepols (sous presse).

Chitty 1966 = Barsanuphius and John, Questions and Answers, ed. Derwas James Chitty (Patrologia orientalis 31/3), Paris: Firmin-Didot, 1966.

Chrysos 2002 = Chrysos, Evangelos, "Illuminating Darkness by Candlelight: Literature in the Dark Ages”, in: P. Odorico - P.A. Agapitos (eds.), Pour une «nouvelle » histoire de la littérature byzantine. Problèmes, méthodes, approches, propositions. Actes du Colloque international philologique Nicosie - Chypre 25-28 mai 2000 (Dossiers byzantins 1), Paris: Centre d'études byzantines, néo-helléniques et sud-est europénnes, EHESS, 2002, 13-24.

Congourdeau 2005 = Congourdeau, Marie-Hélène, « Médecine et théologie chez Anastase le Sinaïte, médecin, moine et didascale », in: V. Boudon-Millot - B. Pouderon (eds.), Les Pères de l'Église face à la science médicale de leur temps (Théologie historique 117), Paris: Beauchesne, 2005, 287-297.

Constas $1999=$ Constas, Nicholas, "Word and Image in Byzantine Iconoclasm. The Biblical Exegesis of Photius of Constantinople", in: Rodney L. Petersen (ed.), The Contentious Triangle: Church, State, and University. A Festschrift in Honor of Professor George Huntston Williams (Sixteenth-Century Essays \& Studies 51), Kirksville, Mo.: Thomas Jefferson Univ. Press, 1999, 97-109.

CPG = Geerard, Maurits, Clavis Patrorum Graecorum, 5 vol., Turnhout: Brepols, 1974-1987; Geerard, Maurits - Noret, Jacques, Supplementum, Turnhout: Brepols, 1998; Noret, Jacques, Addenda vol. III, Turnhout: Brepols, 2003.

Dagron 1981 = Dagron, Gilbert, «Le saint, le savant, l'astrologue. Étude de thèmes hagiographiques à travers quelques recueils de «Questions et réponses» des $\mathrm{V}^{\mathrm{e}}-\mathrm{VII} \mathrm{I}^{\mathrm{e}}$ siècles », in: Hagiographies, cultures et sociétés (IVe-XIIe s.), Paris: Études Augustiniennes, 1981, 143-156.

De Groote 2015 = De Groote, Marc, "The Soterios Project revisited: status quaestionis and the future edition”, Byzantinische Zeitschrift 108 (2015), 63-78.

De Leemans - Goyens 2006 = De Leemans, Pieter - Goyens, Michèle (eds.), Aristotle's Problemata in Different Times and Tongues, Leuven: Leuven University Press, 2006.

Demetracopoulos 2012 = Demetracopoulos, John A., "Thomas Aquinas' Impact on Late Byzantine Theology and Philosophy: The Issues of Method or 'Modus Sciendi' and 'Dignitas Hominis"', in: Andreas Speer - Philipp Steinkrüger (eds.), Knotenpunkt Byzanz: Wissensformen und kulturelle Wechselbeziehungen (Miscellanea mediaevalia 36), Berlin: de Gruyter, 2012, 333-410. 
De Ridder - Levrie sous presse = De Ridder, Eva - Levrie, Katrien, "Capita literature in Byzantium", in: Ceulemans - De Leemans sous presse.

Durst 1989 = Durst, Michael, "Hegesipps Hypomnemata: Titel oder Gattungsbezeichnung? Untersuchungen zum literarischen Gebrauch von Hypomnema - Hypomnemata", Römische Quartalschrift für christliche Altertumskunde und Kirchengeschichte 84 (1989), 299-330.

De Vos sous presse = De Vos, Ilse, "The Manuscript Tradition of the Quaestiones ad Antiochum ducem", in: Ceulemans - De Leemans sous presse.

Gribomont 2012 = Gribomont, Aurélie, « La question du titre dans la littérature byzantine. Quelques pistes de réflexion autour du terme ú ó $\mu \nu \eta \mu \alpha »$, Byzantion 82 (2012), 89-112.

Haldon 1992 = Haldon, John, "The Works of Anastasius of Sinai: A Key Source for the History of Seventh-Century East Mediterranean Society and Belief", in: Cameron - Conrad 1992, 107-147.

Hatlie 1996 = Hatlie, Peter, "Redeeming Byzantine Epistolography", Byzantine and Modern Greek Studies 20 (1996), 213-248.

Heinrici, 1910 = Heinrici, Carl Friedrich Georg, "Zur patristischen Aporienliteratur", Abhandlungen der Sächsischen Akademie der Wissenschaften zu Leipzig, philologisch-historische Klasse, 27/24 (1910).

Heinrici 1911 = Heinrici, Carl Friedrich Georg, "Griechisch-Byzantinische Gesprächsbücher und Verwandtes aus Sammelhandschriften", Abhandlungen der Sächsischen Akademie der Wissenschaften zu Leipzig, philologisch-historische Klasse, 28/8 (1911).

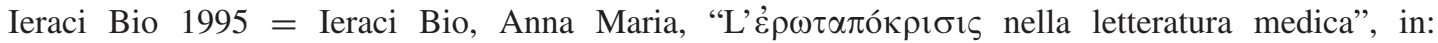
C. Moreschini (ed.), Esegesi, parafrasi e compilazione in età tardoantica: Atti del terzo Convegno dell'Associazione di studi tardoantichi, Napoli: D'Auria, 1995, 187-207.

Johnson 2006 = Johnson, Scott Fitzgerald (ed.), Greek literature in Late Antiquity: dynamism, didacticism, classicism, Aldershot: Ashgate, 2006.

Kiapidou 2013 = Kiapidou, Eirini-Sophia, "Chapters, epistolary essays and epistles. The case of Michael Glykas' Collection of ninety-five texts in the 12th century", Parekbolai 3 (2013), 45-64.

Krausmüller 2013 = Krausmüller, Dirk, "At the resurrection we will not recognise one another": Radical devaluation of social relations in the lost model of Anastasius' and Pseudo-Athanasius' Questions and Answers", Byzantion 83 (2013), 201-227.

Lackner 1991 = Lackner, Wolfgang, "Beobachtungen zum Wortschatz des Pseudo-Kaisarios", in: W. Hörandner (ed.), Lexicographica byzantina. Beiträge zum Symposion zur byzantinischen Lexikographie (Byzantina Vindobonensia 20), Wien: Verlag der Österreichischen Akademie der Wissenschaften, 1991, 207-217.

Lim 2008 = Lim, Richard, "Christians, dialogues and patterns of sociability in late antiquity", in: S. Goldhill (ed.), The End of Dialogue in Antiquity, Cambridge: Cambridge University Press, 2008, 151-172.

Macé 2013 = Macé, Caroline, "Les Quaestiones ad Antiochum ducem d'un Pseudo-Athanase (CPG 2257). Un état de la question”, in: Bussières, 2013, 121-150.

Macé 2014 = Macé, Caroline, «Un fragment Contre les astronomes attribué à Grégoire de Nazianze ou à Grégoire de Nysse (CPG 3063) », in: G. Van Riel - P. d'Hoine (eds.), Fate, Providence and Moral Responsibility. Collected studies in honour of Carlos Steel, Leuven: Leuven University Press, 2014, 357-382.

Macé - De Vos 2013 = Macé, Caroline - Ilse De Vos, "Pseudo-Athanasius, Quaestio ad Antiochum 136 and the Theosophia", in: Studia Patristica LXVI: Papers presented at the $16^{\text {th }}$ International Conference on Patristic Studies held in Oxford 2011, ed. by M. Vinzent, Leuven: Peeters Publishers, 2013, 319-332.

Mimouni 1997 = Mimouni, Simon, «L'Hypomnesticon de Joseph de Tibériade: une œuvre du IV siècle ? », in: Studia Patristica XXXII. Twelfth International Conference on Patristic Studies, Oxford 21-26 August 1995, Leuven: Peeters, 1997, 346-357, repr. in: Mimouni, Simon, Les traditions 
anciennes sur la Dormition et l'Assomption de Marie. Études littéraires, historiques et doctrinales, Leiden: Brill, 2010, 257-273.

Mullett 1992 = Mullett, Margaret, "The Madness of Genre”, Dumbarton Oaks Papers 46 (1992), $233-243$.

Mullett 2008 = Mullett, Margaret, "Epistolography", in: R. Cormack - J. F. Haldon - E. Jeffreys (eds.), The Oxford Handbook of Byzantine Studies, Oxford: Oxford University Press, 2008, 882-892.

Munitiz 2006 = Anastasii Sinaitae Quaestiones et responsiones, ed. Marcel Richard ( $\dagger$ ) et Joseph A. Munitiz (Corpus Christianorum Series Graeca 59), Turnhout: Brepols, 2006.

Odorico 1990 = Odorico, Paolo, "La cultura della $\Sigma v \lambda \lambda o \gamma \eta$. 1) Il cosidetto enciclopedismo bizantino.

2) Le tavole del sapere di Giovanni Damasceno, Byzantinische Zeitschrift, 83 (1990), 1-21.

Odorico 2011 = Odorico, Paolo, « Cadre d'exposition / cadre de pensée : la culture du recueil », in: P. Van Deun - C. Macé (eds.), Encyclopedic Trends in Byzantium? (Orientalia Lovaniensia Analecta 212), Leuven: Peeters, 2011, 89-107.

Papadogiannakis 2006 = Papadogiannakis, Yannis, "Instruction by question and answer in Late Antiquity: the case of Late Antique Erotapokriseis", in: Johnson, 2006, 91-105.

Papadogiannakis $2008=$ Papadogiannakis, Yannis, "Defining Orthodoxy in Pseudo-Justin's Quaestiones et Responsiones ad Orthodoxos", in: H. Zellentin - E. Iricinschki (eds.), Heresy and Identity in Late Antiquity, Tübingen: Mohr Siebeck, 2008, 115-127.

Papadogiannakis 2011 = Papadogiannakis, Yannis, "Encyclopedism' in the Byzantine question-andanswer literature: the case of Pseudo-Kaisarios”, in: P. Van Deun - C. Macé (eds.), Encyclopaedic trends in Byzantium? Proceedings of the International Conference held in Leuven, 6-8 May 2009 (Orientalia Lovaniensia Analecta 212), Leuven: Peeters 2011, 29-44.

Papadogiannakis 2013 = Papadogiannakis, Yannis, "Didacticism, Exegesis and Polemics in PseudoKaisarios's Erotapokriseis”, in: Bussières, 2013, 271-289.

Papadogiannakis 2014 = Papadogiannakis, Yannis, "Individuality and the Resurrection in some Late Antique Texts", in: Johannes Zachhuber - Alexis Torrance (eds.), Individuality in Late Antiquity (Ashgate Studies in Philosophy and Theology in Late Antiquity), Aldershot: Ashgate 2014, 147-166. Papaioannou 2010 = Papaioannou, Stratis, "Letter-Writing", in: P. Stephenson (ed.), The Byzantine World, London - New York: Routledge, 2010, 188-199.

Perczel 2006 = Perczel, Istvan, "Finding a Place for the Erotapokriseis of Pseudo-Caesarius: a New Documents of Sixth-Century Palestinian Origenism", ARAM Periodical 18-19 (2006-2007), 49-83.

PG = Migne, Jacques Paul (ed.) Patrologiae Cursus Completus, Series Graeca, 161 vols., Paris 1857-1866.

Pouderon 2005 = Pouderon, Bernard «Le Pseudo-Justin : ou la constitution d'un corpus apologétique pseudépigraphe », in: Sylvie Crogiez-Pétrequin (ed.), Dieux et hommes: histoire et iconographie des sociétés païennes et chrétiennes de l'Antiquité à nos jours. Mélanges en l'honneur de Françoise Thelamon, Publications de l'Université de Rouen et du Havre, 2005, 49-67.

Rey 2004 = Rey, Alain-Louis, «Les erotapokriseis dans le monde byzantin: Tradition manuscrite des textes anciens et production de nouveaux textes », in: Volgers - Zamagni, 2004, 165-180.

Riedinger 1969 = Riedinger, Rudolf, Pseudo-Kaisarios: Überlieferungsgeschichte und Verfasserfrage (Byzantinisches Archiv 12), München: Beck, 1969.

Riedinger 1989 = Pseudo-Kaisarios, Die Erotapokriseis, ed. Rudolf Riedinger (Die griechischen christlichen Schriftsteller der ersten Jahrhunderte [58]), Berlin: de Gruyter, 1989.

Thomson 2014 = Thomson, Francis, "Byzantine erotapocritic literature in Slavonic translation with special attention to the important role played by Anastasius Sinaita's Interrogationes et Responsiones in the conversion of the Slavs", Byzantion 84 (2014), 385-432.

Tzamalikos 2012 = P. Tzamalikos, Panayiotis, A newly discovered Greek Father: Cassian the Sabaite eclipsed by John Cassian of Marseilles (Supplements to Vigiliae Christianae 111), Leiden: Brill, 2012. 
van der Horst 2004 = van der Horst, P.W., "Philo and the Rabbis on Genesis: Similar Questions, Different Answers", in: Volgers - Zamagni, 2004, 55-70.

Volgers - Zamagni 2004 = Volgers, Annelie - Zamagni, Claudio (eds.), Early Christian Question-andAnswer Literature in Context. Proceedings of the Utrecht Colloquium, 13-14 October 2003, Leuven - Paris - Dudley, MA: Peeters, 2004.

Zamagni 2004 = Zamagni, Claudio, « Une introduction méthodologique à la littérature patristique des questions et réponses : le cas d'Eusèbe de Césarée », in: Volgers - Zamagni, 2004, 7-24.

Zamagni 2013 = Zamagni, Claudio, "Is the Question-and-Answer Literary Genre in Early Christian Literature an Homogeneous Group?”, in: Bussières, 2013, 241-267. 